\title{
SARCOMA FIBROMIXÓIDE DE BAIXO GRAU DA PAREDE TORÁCICA: RELATO DE CASO*
}

\author{
Emerson L. Gasparetto ${ }^{1}$, Cesar Inoue ${ }^{2}$, Sérgio E. Ono ${ }^{1}$, Andresa Braga ${ }^{1}$, Gabriela de Melo Rocha ${ }^{2}$, \\ Dante L. Escuissato ${ }^{3}$, Edson Marchiori ${ }^{4}$
}

\begin{abstract}
Resumo Este relato descreve um caso de sarcoma fibromixóide de baixo grau na parede torácica em uma paciente feminina de 23 anos de idade. A radiografia de tórax e a tomografia computadorizada demonstraram massa heterogênea na região inferior do hemitórax direito, com necrose e focos de calcificação. 0 exame histológico foi sugestivo de leiomioma, mas a imuno-histoquímica definiu o diagnóstico de sarcoma fibromixóide de baixo grau. A evolução clínica do caso foi boa, apesar dos aspectos de malignidade demonstrados na tomografia computadorizada.

Unitermos: Tomografia computadorizada; Parede torácica; Sarcoma fibromixóide.
\end{abstract}

Abstract Low-grade fibromyxoid sarcoma of the chest wall: a case report.

We report the case of a 23-year-old female patient with a low-grade fibromyxoid sarcoma involving the chest wall. The chest radiography and computed tomography scan showed a heterogeneous mass in the lower right hemithorax, with necrosis and calcification foci. Histological examination was suggestive of a leiomyoma but the immunohistochemical study proved to be a low-grade fibromyxoid sarcoma. The clinical outcome of this patient was good, although the computed tomography scan showed signs of malignancy.

Key words: Computed tomography; Chest wall; Fibromyxoid sarcoma.

\section{INTRODUÇÃO}

O sarcoma fibromixóide de baixo grau é uma neoplasia de tecidos moles com aspecto benigno caracterizada por recidivas locais ao longo dos anos.

Este tumor foi descrito por Evans em 1987, o qual apresentou dois casos de tumores metastáticos de tecidos moles com aparência histológica benigna. Esta neoplasia afeta adultos jovens e apresenta predileção por sítios profundos, incluindo coxa, região inguinal, ombro, axila, períneo, pescoço, nádegas, retroperitônio e fossa poplítea, sendo raro em outras localizações $^{(\mathbf{1})}$. O exame histológico demonstra áreas fibróides e mixóides de tamanhos variáveis, discreta a moderada celularidade

* Trabalho realizado na Disciplina de Radiologia Médica do Departamento de Clínica Médica da Universidade Federal do Paraná (UFPR), Curitiba, PR.

1. Médicos Residentes do Hospital de Clínicas da UFPR.

2. Acadêmicos de Medicina da UFPR.

3. Professor Assistente da Disciplina de Radiologia Médica da UFPR.

4. Professor Titular de Radiologia da Universidade Federa Fluminense (UFF), Coordenador Adjunto do Curso de Pós-Graduação da Universidade Federal do Rio de Janeiro (UFRJ).

Endereço para correspondência: Dr. Emerson L. Gasparetto. Serviço de Radiologia Médica, Hospital de Clínicas - UFPR. Rua General Carneiro, 181. Curitiba, PR, 80060-900. E-mail: gasparetto@hotmail.com

Recebido para publicação em 28/10/2004. Aceito, após revisão, em 15/12/2004. e células fibroblásticas fusiformes, com ou sem pleomorfismo nuclear e poucas figuras mitóticas ${ }^{(2)}$. O exame macroscópico em geral demonstra massas grandes com margens bem definidas ${ }^{(3)}$.

Aos exames de imagem, os sarcomas de tecidos moles tipicamente aparecem como lesões com densidade de tecidos moles ${ }^{(\mathbf{4})}$. Embora sarcomas primários torácicos comumente se manifestem como massas grandes e heterogêneas, estes tumores podem ter apresentações radiológicas variadas, como nódulos pulmonares solitários, tumores endobrônquicos centrais e massas intraluminais nas artérias pulmonares ${ }^{(\mathbf{5})}$.

Neste trabalho é descrito um caso de sarcoma fibromixóide de baixo grau da parede torácica, enfatizando-se os aspectos radiológicos e histológicos deste tumor.

\section{RELATO DO CASO}

Paciente do sexo feminino, 23 anos de idade, há dois anos com dor na região toracolombar direita. Duas semanas antes da internação a paciente apresentou dispnéia e tosse produtiva. $\mathrm{O}$ exame físico revelou redução dos ruídos pulmonares à direita.

A radiografia de tórax demonstrou opacificação dos dois terços inferiores do hemitórax direito, com desvio das estruturas mediastinais para a esquerda e destruição do terço proximal da décima costela direita (Figura 1). A tomografia computadorizada demonstrou volumosa massa heterogênea ocupando os dois terços inferiores do hemitórax direito, com colapso pulmonar adjacente e pequeno derrame pleural associado. A lesão apresentava áreas hipodensas (necrose) e focos de calcificação em seu interior. Além disso, observava-se destruição do décimo arco costal e desvio do mediastino e do coração para a esquerda (Figura 2).

A biópsia da massa revelou neoplasia constituída por células fusiformes com citoplasma eosinofílico e núcleos hipercrômicos, vesiculados e alongados. O tumor apresentava áreas fibróides e áreas de vascularização irregulares, mostrando um padrão "hemagiopericitoma-like". Focos de estroma mixóide também foram visualizados, além de histiócitos e linfócitos entre as células neoplásicas. Foi definido o diagnóstico de tumor mesenquimal de baixo grau e a paciente foi submetida à ressecção cirúrgica da lesão. Durante o procedimento cirúrgico evidenciou-se que o tumor se originava na parede torácica e a lesão foi totalmente ressecada.

Onze meses após a primeira cirurgia a paciente apresentou recidiva local, sendo 


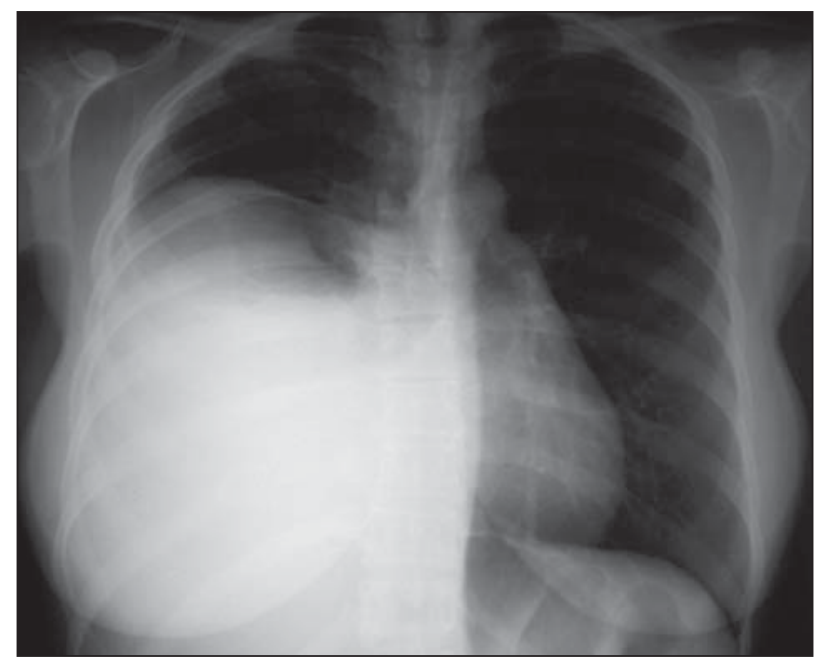

A

Figura 1. Em A, radiografia de tórax em póstero-anterior demonstra opacificação dos dois terços inferiores do hemitórax direito, com desvio das estruturas mediastinais para a esquerda. Em B, detalhe da radiografia evidencia a destruição do terço proximal da décima costela direita.

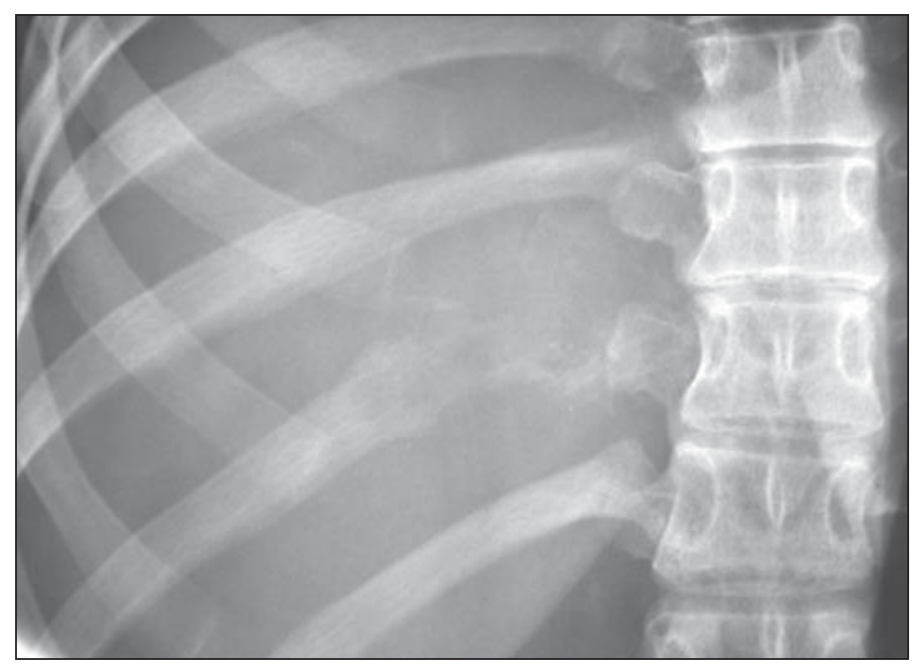

B

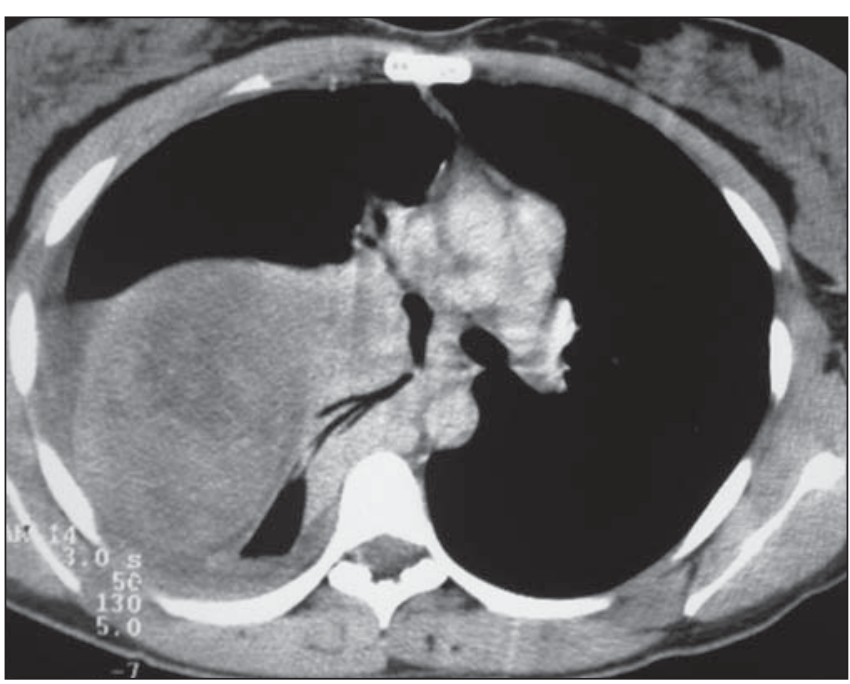

A

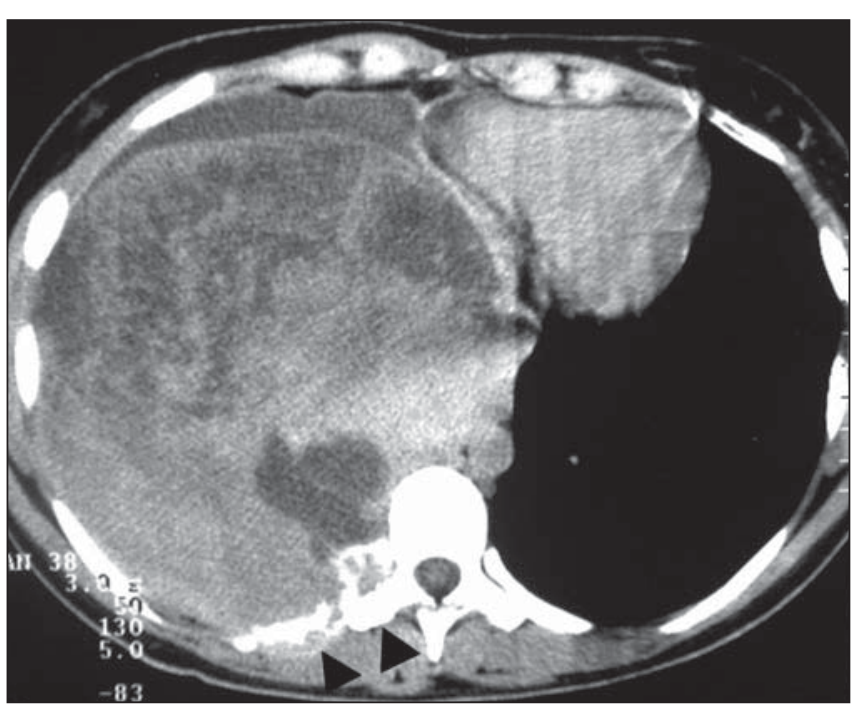

C

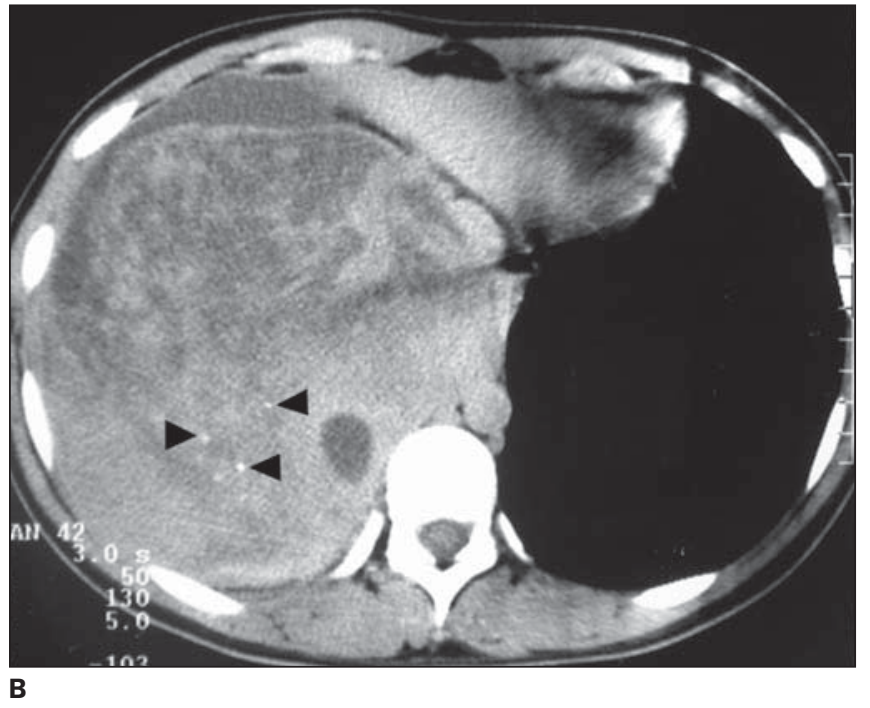

Figura 2. Em A, tomografia computadorizada demonstra massa heterogênea no hemitórax direito, associada a pequeno derrame pleural e atelectasia do lobo médio. Em B, a tomografia computadorizada evidencia pequenas calcificações no interior da lesão (setas) e compressão sobre o mediastino. Em C, observamse destruição da porção proximal do décimo arco costal direito e compressão sobre o coração. 
realizada nova ressecção cirúrgica. O estudo histológico demonstrou áreas fibróides, bem como uma área focal de proliferação de células fusiformes sugestiva de leiomioma. Desta vez foi realizado estudo imuno-histoquímico pelo método da avidina-biotina peroxidase, com controles positivos e negativos. Foram utilizados os seguintes anticorpos para detecção do antígeno: actina HHF-35, desmina, proteína $S$ 100, CD-34 e vimentina. Os resultados foram: actina HHF-35, desmina e vimentina positivos, e proteína S-100 e CD-34 negativos. Os aspectos histológicos e o perfil imuno-histoquímico definiram o diagnóstico de sarcoma fibromixóide de baixo grau de malignidade.

Atualmente, 12 meses após a segunda cirurgia, a paciente não apresenta evidências clínicas ou radiológicas de recidiva tumoral.

\section{DISCUSSÃO}

Os sarcomas primários do tórax são raros. Os critérios diagnósticos ainda não estão totalmente definidos, sendo estabelecido este diagnóstico apenas após a exclusão de doença metastática e neoplasias pulmonares primárias. Usualmente, estes tumores afetam adultos jovens entre a terceira e quinta décadas de vida, com discreto predomínio pelo sexo masculino ${ }^{(6)}$. $\mathrm{O}$ diagnóstico diferencial inclui tumores benignos (neurofibroma mixóide e fibromatose desmóide), tumores de malignidade intermediária (dermatofibrossarcoma protuberans) e outros sarcomas (mixofibrossarcoma de baixo grau, lipossarcoma de células fusiformes $)^{(7)}$. Os diferentes tipos histológicos de sarcoma são indistinguíveis pelos métodos de imagem. Contudo, dife- renças no quadro clínico e na localização do tumor, além de achados como calcificações e envolvimento de arcos costais, podem ser úteis na tentativa de se estabelecer o diagnóstico diferencial acurado ${ }^{(5)}$.

O sarcoma fibromixóide de baixo grau foi descrito primeiramente por Evans em 1987, o qual apresentou dois casos de tumores de partes moles com comportamento indolente, aparência histológica benigna, mas com surgimento de metástases na evolução $^{(\mathbf{1})}$. Em 1993, o mesmo autor publicou uma série de 12 casos, enfatizando o comportamento clínico agressivo desta neoplasia ${ }^{(2)}$. Histologicamente, o sarcoma fibromixóide de baixo grau é caracterizado pela presença de células fusiformes, mostrando arranjo linear focal ou não, e alternando áreas de estroma fibróide e mixóide ${ }^{(\mathbf{7 , 8})}$. O tumor descrito neste relato preencheu os critérios estabelecidos por Evans para o diagnóstico de sarcoma fibromixóide de baixo grau.

Um diagnóstico diferencial de importância clínica é o mixofibrossarcoma de baixo grau. Este tumor, ao contrário do sarcoma fibromixóide de baixo grau, é diagnosticado em pacientes idosos, em geral acometendo tecido celular subcutâneo, e mostrando mais atipia nuclear com aneuploidia. O mixofibrossarcoma de baixo grau é uma neoplasia uniformemente mixóide, sem áreas de estroma colagenoso. Este aspecto histológico é importante na diferenciação destes dois tumores, já que, ao contrário do sarcoma fibromixóide de baixo grau, o mixofibrossarcoma de baixo grau raramente apresenta metástases ${ }^{(\mathbf{6})}$.

De acordo com a terapêutica estabelecida no presente caso, o tratamento de escolha é a ressecção cirúrgica do tumor primário, das recidivas e das metástases. Qui- mioterapia e radioterapia parecem não mudar o curso da doença ${ }^{(6)}$. A evolução do tumor é imprevisível, sendo que em geral segue um curso indolente, mas maligno, com recidivas freqüentes, sendo na maioria dos casos em menos de dois anos ${ }^{(2,7)}$. A paciente deste relato apresentou recidiva tumoral 11 meses após a primeira ressecção cirúrgica.

No presente estudo não observamos padrões de imagem que auxiliem no diagnóstico diferencial entre o sarcoma fibromixóide de baixo grau e as demais neoplasias que acometem a parede torácica ou outras estruturas do tórax. A definição histológica deste tumor é importante, já que, apesar de indolente, seu curso clínico pode ser complicado por recidivas locais e/ou metástases.

\section{REFERÊNCIAS}

1. Evans HL. Low-grade fibromyxoid sarcoma. A report of two metastasizing neoplasms having a deceptively benign appearance. Am J Clin Pathol 1987;88:615-619.

2. Evans HL. Low-grade fibromyxoid sarcoma. A report of 12 cases. Am J Surg Pathol 1993;17:595600 .

3. Takanami I, Takeuchi K, Naruke M. Low-grade fibromyxoid sarcoma arising in the mediastinum. J Thorac Cardiovasc Surg 1999;118:970-971.

4. Jeung MY, Gangi A, Gasser B, et al. Imaging of chest wall disorders. RadioGraphics 1999;19:617637.

5. Gladish GW, Sabloff BM, Munden RF, Truong MT, Erasmus JJ, Chasen MH. Primary thoracic sarcomas. RadioGraphics 2002;22:621-637.

6. Shidham VB, Ayala GE, Lahaniatis JE, Garcia FU. Low-grade fibromyxoid sarcoma: clinicopathologic case report with review of the literature. Am J Clin Oncol 1999;22:150-155.

7. Goodlad JR, Mentzel T, Fletcher CDM. Low grade fibromyxoid sarcoma: clinicopathological analysis of eleven new cases in support of a distinct entity. Histopathology 1995;26:229-237.

8. Dvornik G, Barbareschi M, Gallotta P, Dalla Palma P. Low grade fibromyxoid sarcoma. Histopathology 1997;30:274-276. 